\title{
Design of Reverse Curves Adapted to the Satellite Measurements
}

\author{
Wladyslaw Koc \\ Faculty of Civil and Environmental Engineering, Gdańsk University of Technology, Narutowicza 11/12, 80-233 Gdańsk, Poland \\ Correspondence should be addressed to Wladyslaw Koc; kocwl@pg.gda.pl
}

Received 26 November 2015; Revised 25 February 2016; Accepted 13 March 2016

Academic Editor: Samer Madanat

Copyright (C) 2016 Wladyslaw Koc. This is an open access article distributed under the Creative Commons Attribution License, which permits unrestricted use, distribution, and reproduction in any medium, provided the original work is properly cited.

\begin{abstract}
The paper presents a new method for designing railway route in the direction change area adapted to the Mobile Satellite Measurements technique. The method may be particularly useful in the situations when both tangents cannot be connected in an elementary way using a circular arc with transition curves. Thus, the only solution would be the application of two circular arcs of opposite curvature signs, that is, the use of an inverse curve. It has been assumed that the design of the geometrical layout will take place within an adequate local coordinate system. The solution of the design problem takes advantage of a mathematical notation and concentrates on the determination of universal equations describing the entire geometrical layout. This is a sequential operation involving successive parts of the mentioned layout. This universal algorithm can be easily applied to the computer software which will allow generating, in an automatic way, other geometrical layouts. Then, the choice of the most beneficial variant from the point of obtained trains velocities while minimizing the track axis offsets will be held using the optimization techniques. The current designing methods do not provide such opportunities. The presented method has been illustrated by appropriate calculation examples.
\end{abstract}

\section{Introduction}

An inspiration for undertaking the analyzed problem is undoubtedly the new technology-the application to the railway track satellite measurements GPS. The global positioning system GPS [1-5] enables determining the coordinates of points in a uniform three-dimensional reference system WGS 84 whose origin is placed in the centre of the Earth's mass. Effective measurements of railway track might be obtained by the method of Mobile Satellite Measurements elaborated by a scientific team of the Gdańsk University of Technology and the Naval Academy in Gdynia [6,7]. This method refers to a pilot study [8] and involves driving through the tested section of the route being inspected by the use of antennas installed on a travelling rail carriage.

The Mobile Satellite Measurements make it possible to determine the coordinates of the existing railway route using the Cartesian coordinate system (which in Poland corresponds to the national spatial reference system [9]) as described in the works [10-13]. It would be advantageous if the newly designed track axis coordinates were determined in mentioned system; in particular, the coordinates are used in setting out the route in terrain.
General principles for design of track geometry were formulated in the nineteenth century. Then their continuous modification followed, which was reflected in the constantly changing regulations. It should be noted, however, that the development of computer technology constantly stimulates the ongoing search for new solutions, presented among others in [14-17].

The significant precision obtained in the method of Mobile Satellite Measurements in terms of the determination of coordinates in horizontal plane (with an error in the range of several millimeters) [12] inclines to work out new design methods adapted to the satellite measurement technique [18, $19]$ and to new computer-aided programs [20].

The interest of analyzed problem is confirmed by the investigations carried out in Europe in 2006-2010 as part of the INNOTRACK program [21]. The investigations were coordinated by International Union of Railways (UIC). Over 30 participants were engaged in the project including 8 leading infrastructure managing directors (among others from UK, Germany, and France). It appears that on the list of the most frequent problems raised by the infrastructure board of directors is the ascertainment "bad track geometry." The methods of geometric shaping of tracks that have been 
used so far proved to be ineffective. Thus, there is plenty of work to do to improve the present unfavorable situation.

The difficulties connected with the geometric track shaping in horizontal plane result from the fact that the applied geometric elements, like straight sections, radii of circular arcs, and transition curves, are very often characterized by large lengths and therefore a visual evaluation of the whole system using the traditional geodesic techniques becomes ineffective. The system should be divided into parts and analyzed individually, which causes extra errors.

At this point it is essential to note a very important fact. The reason is that the railway project has its own characteristics, which is in fact generally connected with existing layout and the regulation of the track axis. In situations relating to regions requiring an alternative route direction, the design will, in principle, be based on making a correction of the circular arc radius as well as the type and length of the transition curves so that the new geometric layout is most desirable from the rail vehicles kinematic point of view. Simultaneously, its position in horizontal plane will not divert too much from the existing layout.

Determining the values of geometrical parameters which will ensure meeting these conditions becomes in fact a key issue. Specifying these parameters requires consideration of multiple design options and making appropriate choice, using optimization techniques. The new method of calculating the coordinates adapted to the satellite measurements is essential to generate variants of railway geometrical layout. Only after obtaining the appropriate values of the geometric parameters as a result of this procedure it is possible to use in a rational way any of the commercial computer programs supporting the designing process.

As proved by the satellite measurements that have been carried out so far, the shape of the railway track in operation can be so deformed that the determination of the main directions turns out to be impossible; one cannot apply a model system to the design: transition curve-circular arctransition curve. The only solution in this case is to introduce two circular arcs of different radius to the geometrical layout, which means applying a compound curve [19].

All this, however, does not cover the whole problem yet. But under some conditions even the use of a compound curve does not allow us to connect the major directions of the route. In consequence, it is necessary to use two inverse arcs in the geometrical layout. A description of the design procedure for this type of situation can be found in the content of the paper. The presented conception of the designing technique related to a route realignment area creates an opportunity, like other elaborated methods, to obtain an analytical solution by the application of adequate mathematical formulae, which is more convenient for practical usage.

\section{General Assumptions}

The route major directions in the Polish National Spatial Reference System can be defined by the following equations [9]:

Straight 1:

$$
X_{1}=A_{1}+B_{1} Y .
$$

Straight 2:

$$
X_{2}=A_{2}+B_{2} Y .
$$

In the above equations, $A_{1}$ and $A_{2}$ are absolute terms in the expression and $B_{1}$ and $B_{2}$ define the slope coefficients of the two straights. The straights have similar slope coefficient values and intersect at a distant point (they can also run in parallel to each other). Such a situation justifies the connection of both straights by reverse curve.

The designing procedure will take place within an appropriate local system of coordinates (LCS), making it possible to present the course of the route in functional notation. This system results from the adoption of the coordinates of its starting point $O\left(Y_{O}, X_{O}\right)$ on Straight 1 and making a rotation of $\beta$ angle. The problem of obtaining appropriate inclinations of Straights 1 and 2 in LCS system becomes a crucial question in this matter. The inclinations must be positive to operate the positive values of ordinates $y(x)$ and also advantageous in the view of the procedure of determining these ordinates, that is, neither too big nor too small. In this respect the $\beta$ rotation angle will play a decisive role in it.

An assumption is made that, within system $x, y$, Straight 1 will pass through the centre of the system with a slope angle being equal to $\pi / 4$. For the reason that Straight 2 inclination value is similar to Straight 1 it will certainly be placed within interval $(0, \pi / 2)$, closer to the centre of that (i.e., inclination of Straight 1) than to its boundaries. A positive inclination of both straights ensures an analytical description of the whole geometrical layout by the use of explicit functions $y(x)$ related to the circular arcs.

In order to insert an inverse curve between both straights it is necessary to find such coordinates of point $O\left(Y_{O}, X_{O}\right)$, where Straight 2 can be placed on the right side of Straight 1. Knowledge of the point $O\left(Y_{O}, X_{O}\right)$ coordinates and the rotation angle $\beta$ enables mutual points transformation between global and local coordinate system. The value of turning angle $\beta$ is determined by the use of the following equation:

$$
\beta=\varphi_{1}-\frac{\pi}{4},
$$

where $\varphi_{1}=\operatorname{atan} B_{1}$ for $B_{1}>0$ and $\varphi_{1}=\operatorname{atan} B_{1}+\pi$ for $B_{1}<0$. To obtain a positive value of angle $\beta$ from the above formula, the left turn of the system should be made, whereas, in the case of a negative magnitude, a right turn of the system should be made.

In assuming the coordinates of point $O\left(Y_{O}, X_{O}\right)$ along Straight 1 and determining the turning angle $\beta$, it is possible to make a transformation of Straights 1 and 2 to the local coordinate system $x, y$. The entire geometric system in LCS system under consideration is presented in Figure 1.

The position of an arbitrary point along the route in local system $x, y$ can be obtained using the following equations [22]:

$$
\begin{aligned}
& x=\left(Y-Y_{O}\right) \cos \beta+\left(X-X_{O}\right) \sin \beta, \\
& y=-\left(Y-Y_{O}\right) \sin \beta+\left(X-X_{O}\right) \cos \beta .
\end{aligned}
$$




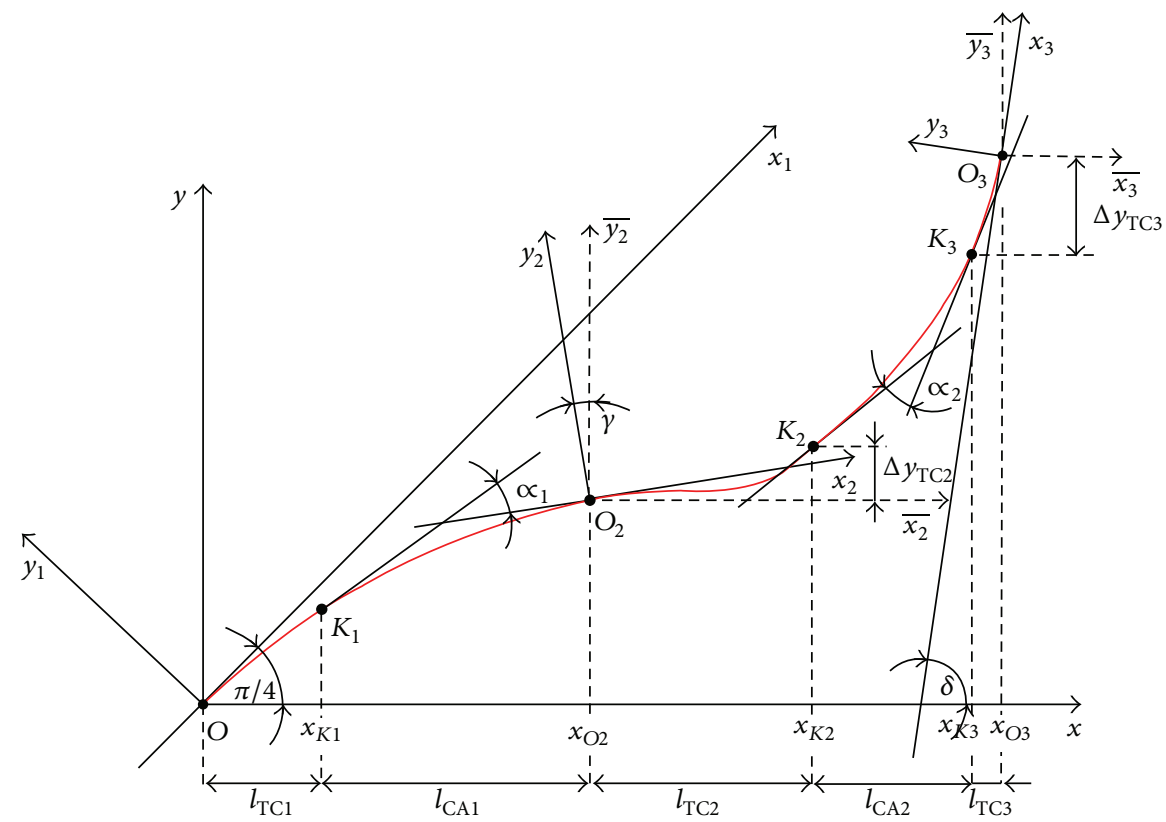

FIGURE 1: The geometrical layout under consideration in the local coordinate system.

In terms of the local coordinate system Straight 1 is described by

$$
y_{(1)}(x)=x \text {. }
$$

A general notation of Straight 2 takes the following form:

$$
y_{(2)}(x)=a_{2}+b_{2} x
$$

where

$$
\begin{aligned}
a_{2} & =-\left(\frac{X_{O}-\left[\tan (\beta+\pi / 2) Y_{O}\right]-A_{2}}{B_{2}-[\tan (\beta+\pi / 2)]}-Y_{O}\right) \sin \beta \\
& +\left[\left(A_{2}+B_{2} \frac{X_{O}-\left[\tan (\beta+\pi / 2) Y_{O}\right]-A_{2}}{B_{2}-[\tan (\beta+\pi / 2)]}\right)\right. \\
& \left.-X_{O}\right] \cos \beta, \\
b_{2} & =\tan \delta .
\end{aligned}
$$

Angle $\delta$, determining the slope of Straight 2 to axis $x$ in the LCS system, is obtained from the formula

$$
\delta=\varphi_{2}-\beta,
$$

where $\varphi_{2}=\operatorname{atan} B_{2}$ for $B_{2}>0$ and $\varphi_{2}=\operatorname{atan} B_{2}+\pi$ for $B_{2}<0$.

\section{Choice of Parameters to Design the Geometric System}

The designed geometric system connecting Straight 1 with Straight 2 in the local system of coordinates $x, y$ is composed of the following components (Figure 1): (i) First transition curve (TC1) of a specified type, of length $l_{1}$.

(ii) First circular arc (CA1) of radius $R_{1}$ and length $l_{R 1}$.

(iii) Second transition curve (TC2) of a specified type, of length $l_{2}$

(iv) Second circular arc (CA2) of radius $R_{2}$ and undefined length $l_{R 2}$.

(v) Third transition curve (TC3) of a specified type, of length $l_{3}$.

Values $l_{1}, R_{1}, l_{2}, R_{2}$, and $l_{3}$ result from the speed analysis carried out for the designed system. Value $l_{R 1}$ is, in principle, arbitrary, while $l_{R 2}$ is the final magnitude closing up the whole system. However, one should take into account that the raised problem can be solved when advantage is taken of an appropriate configuration of the abovementioned parameters. A key role in this procedure is the characteristic of the curvature used. Curve TC1 and arc CA1 have a negative curvature, while arc CA2 and curve TC3 have a positive one. Due to this fact the slope angle of tangent on curve TC1 and arc CA1 is decreasing but on arc CA2 and on curve TC 3 it is rising. However, the following condition should be fulfilled (Figure 1):

$$
\frac{\pi}{4}+\Theta\left(l_{1}\right)-\alpha_{1}+\Theta\left(l_{2}\right)+\alpha_{2}-\Theta\left(-l_{3}\right)=\delta,
$$

where $\Theta\left(l_{1}\right)$ is slope angle of tangent at the end of TC1 in $x_{1}$, $y_{1}$ coordinate system, $\alpha_{1}$ is angle of sense CA1, $\Theta\left(l_{2}\right)$ is slope angle of tangent at the end of TC2 in $x_{2}, y_{2}$ coordinate system, $\alpha_{2}$ is angle of sense CA2, and $\Theta\left(-l_{3}\right)$ is slope angle of tangent at the end of TC3 in the $x_{3}, y_{3}$ coordinate system.

The only element causing a diversity of the curvature sign is curve TC2. The curvature along it changes from negative 
to positive. Simultaneously, the value of angle $\Theta\left(l_{2}\right)$ is either positive or negative. In the case of $\delta<\pi / 4$ it would be advantageous if $\Theta\left(l_{2}\right)<0$, which corresponds to the situation when $R_{1}<R_{2}$. When $\delta>\pi / 4$ it would be profitable if $\Theta\left(l_{2}\right)>0$, leading to relation $R_{1}>R_{2}$.

\section{Transition Curve TC1}

The ordinates of transition curve TC1 are determined by the use of auxiliary system of coordinates $x_{1}, y_{1}$ (ACS1) (Figure 1). The course of proceeding takes the following form:

(i) Determination of the type of the transition curve.

(ii) Assumption of the length $l_{1}$ of curve (measured along the curve) and radius $R_{1}$ of the adjacent circular arc CA1.

This in consequence gives the parametric equations: $x_{1}(l)$, $y_{1}(l), l \in\left\langle 0, l_{1}\right\rangle$.

For the reason that regardless of the type of the transition curve connecting the straight with the circular arc $\left|\Theta\left(l_{1}\right)\right|=$ $l_{1} / 2 R_{1}(\mathrm{rad})$, the tangent value at the end of transition curve $\mathrm{TCl}$ is defined by formula

$$
y_{1}^{\prime}\left(l_{1}\right)=-\tan \left(\frac{l_{1}}{2 R_{1}}\right) .
$$

The next step is to transform curve TC1 to the local system of coordinates $x, y$ (Figure 1). This is accomplished by a right turn of the axis of system ACS1 through angle $\pi / 4$. Consequently the following parametric equations are obtained:

$$
\begin{aligned}
& x(l)=x_{1}(l) \cos \frac{\pi}{4}-y_{1}(l) \sin \frac{\pi}{4}, \\
& y(l)=x_{1}(l) \sin \frac{\pi}{4}+y_{1}(l) \cos \frac{\pi}{4},
\end{aligned}
$$

$$
l \in\left\langle 0, l_{1}\right\rangle .
$$

Inserting the final value of parametric $l$ (i.e., $l=l_{1}$ ) into (9) it is possible to obtain coordinates of the end of curve $\mathrm{TCl}$ (i.e., point $\left.K_{1}\right): x_{K 1}=l_{K P 1}$ and $y_{K 1}$. The tangent value $s_{K 1}$ at point $K_{1}$ is

$$
s_{K 1}=\tan \left(-\frac{l_{1}}{2 R_{1}}+\frac{\pi}{4}\right) .
$$

\section{Circular Arc CA1}

The diagram illustrating the position of the circular arc CA1 is shown in Figure 2. Assumption is made of the circular arc length $l_{R 1}$ (measured along the arc). Coordinates of point $S_{1}\left(x_{S 1}, y_{S 1}\right)$, the centre of arc CAl, are determined:

$$
\begin{aligned}
& x_{S 1}=x_{K 1}+\frac{s_{K 1}}{\sqrt{1+s_{K 1}^{2}}} R_{1}, \\
& y_{S 1}=y_{K 1}-\frac{1}{\sqrt{1+s_{K 1}^{2}}} R_{1} .
\end{aligned}
$$

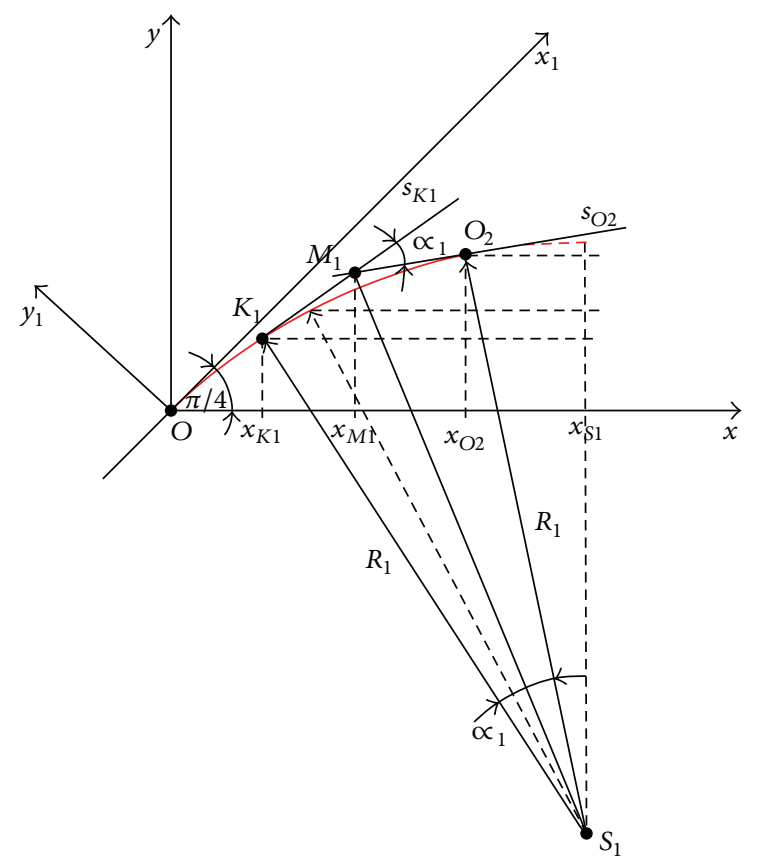

FIGURE 2: Diagram illustrating the position of circular arc CA1.

The equation of circular arc CA1 is as follows:

$$
\begin{aligned}
y(x)_{\mathrm{CA} 1}=y_{S 1}+\left[R_{1}^{2}-\left(x_{S 1}-x\right)^{2}\right]^{1 / 2}, & \\
& x \in\left\langle x_{K 1}, x_{\mathrm{O} 2}\right\rangle .
\end{aligned}
$$

The turning angle of the arc CA1 tangents is

$$
\propto_{1}=\frac{l_{R 1}}{R_{1}} .
$$

Inclination $s_{\mathrm{O} 2}$ of tangent to $\operatorname{arc} \mathrm{CA} 1$ at its end, at point $\mathrm{O}_{2}$, is

$$
s_{O 2}=\tan \left(\operatorname{atan} s_{K 1}-\propto_{1}\right) .
$$

One should now determine the coordinates of point $\mathrm{O}_{2}$, the end of circular arc CA1. For this purpose it is necessary to find the coordinates of point $M_{1}$ (Figure 2). The coordinates of point $\mathrm{O}_{2}$ are as follows:

$$
\begin{aligned}
& x_{\mathrm{O} 2}=x_{M 1}+\frac{\tan \left(\propto_{1} / 2\right)}{\sqrt{1+s_{\mathrm{O} 2}^{2}}} R_{1}, \\
& y_{\mathrm{O} 2}=y_{M 1}+\frac{s_{\mathrm{O} 2} \tan \left(\propto_{1} / 2\right)}{\sqrt{1+s_{\mathrm{O} 2}^{2}}} R_{1} .
\end{aligned}
$$

\section{Transition Curve TC2}

The diagram illustrating the position of transition curve TC2 is given in Figure 3. The ordinates of transition curve TC2 are determined by using an auxiliary system of coordinates $x_{2}, y_{2}$ (ACS2). The transition curve TC2 connects opposite arcs of radii $R_{1}$ and $R_{2}$. 


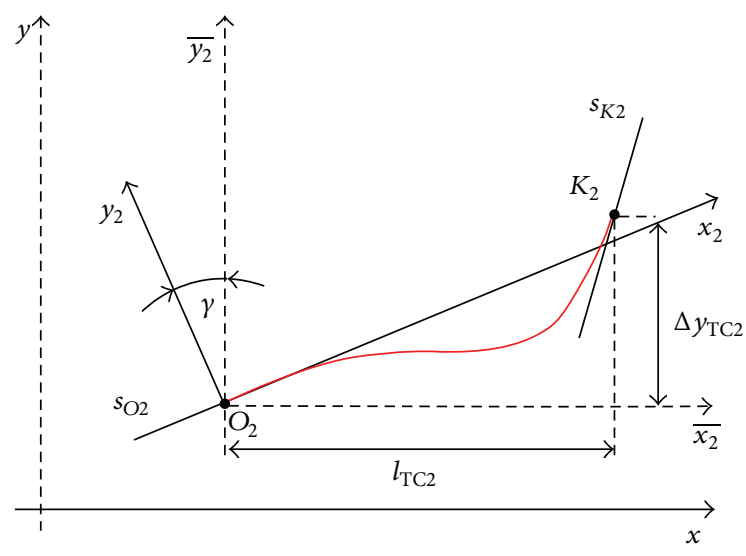

FIGURE 3: Diagram presenting the position of transition curve TC2.

The course of proceeding is as follows:

(i) A linear or nonlinear form of curvature is assumed.

(ii) The length $l_{2}$ of curve (measured along the curve) and the radius $R_{2}$ of the adjacent circular arc CA2 are taken into account.

(iii) Parametric equations $x_{2}(l), y_{2}(l), l \in\left\langle 0, l_{2}\right\rangle$, are obtained.

The examples of solving linear and nonlinear curvature distribution along the length of a curve have been given in the paper [23]. Other forms of formulae are related to inflexion point $l_{0}$ (i.e., for $\left.l \in\left\langle 0, l_{0}\right\rangle\right)$ on the curvature diagram, whereas others refer to the final region $l \in\left\langle l_{0}, l_{2}\right\rangle$.

The next step is the transformation of curve TC2 to the auxiliary coordinate system $\overline{x_{2}}, \overline{y_{2}}(\overline{\mathrm{ACS} 2})$ (Figure 3 ). The position of the system is determined by angle $\gamma=\operatorname{atan} s_{\mathrm{O} 2}$. If the slope $s_{\mathrm{O} 2}=\tan \left(\operatorname{atan} s_{K 1}-\propto_{1}\right)>0$, then it is necessary to make a right turn of system ACS2. However, if the inclination $s_{\mathrm{O} 2}<0$, one should make a left turn of system ACS2. Since angle $\gamma=\left|\operatorname{atan} s_{\mathrm{O} 2}\right| \in\langle 0, \pi / 2\rangle$, the following expressions are obtained:

$$
\begin{array}{r}
x=x_{\mathrm{O} 2}+\overline{x_{2}}(l)=x_{\mathrm{O} 2}+x_{2}(l) \cos \gamma \mp y_{2}(l) \sin \gamma, \\
y=y_{\mathrm{O} 2}+\overline{y_{2}}(l)=y_{\mathrm{O} 2} \pm x_{2}(l) \sin \gamma+y_{2}(l) \cos \gamma, \\
l \in\left\langle 0, l_{2}\right\rangle .
\end{array}
$$

Values $l_{\mathrm{TC} 2}$ and $\Delta y_{\mathrm{TC} 2}$ (Figure 3) are determined from the equations

$$
\begin{aligned}
l_{\mathrm{TC} 2} & =\left|\overline{x_{2}}\left(l_{2}\right)\right|=\left|x_{2}\left(l_{2}\right) \cos \gamma \mp y_{2}\left(l_{2}\right) \sin \gamma\right|, \\
\Delta y_{\mathrm{TC} 2} & =\left|\overline{y_{2}}\left(l_{2}\right)\right|=\left| \pm x_{2}\left(l_{2}\right) \sin \gamma+y_{2}\left(l_{2}\right) \cos \gamma\right| .
\end{aligned}
$$

The coordinates of point $K_{2}$ are as follows:

$$
\begin{aligned}
& x_{K 2}=x_{\mathrm{O} 2}+l_{\mathrm{TC} 2}, \\
& y_{K 2}=y_{\mathrm{O} 2}+\Delta y_{\mathrm{TC} 2},
\end{aligned}
$$

whereas the tangent value $s_{K 2}$ at point $K_{2}$ in $\overline{\mathrm{ACS} 2}$ and LCS system is

$$
s_{K 2}=\tan \left[\Theta\left(l_{2}\right) \pm \gamma\right] .
$$

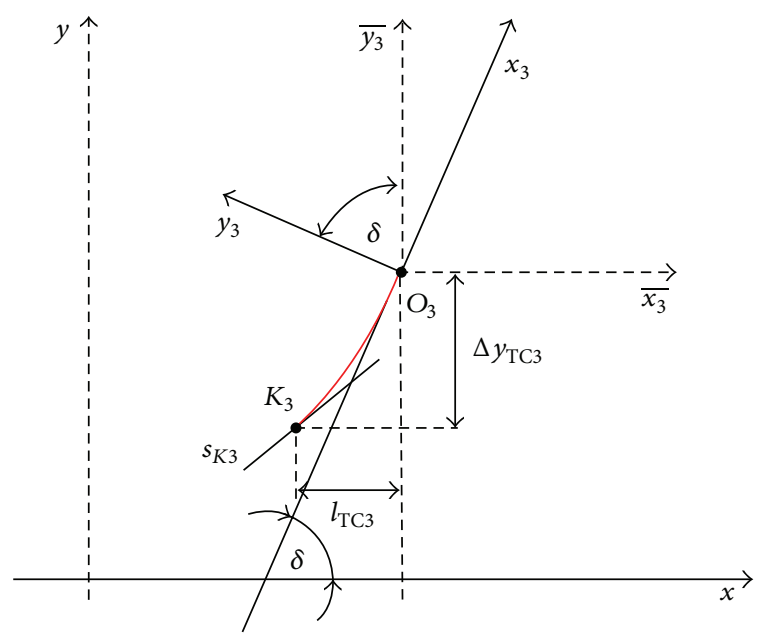

FIGURE 4: Diagram illustrating the position of transition curve TC3.

\section{Transition Curve TC3}

To determine the position of the end of arc CA2 unknown at this stage of investigation, it is necessary to find the value of tangent $s_{K 3}$ at the end of transition curve TC3 being of significance in view of arc CA2. A scheme presenting the position of transition curve TC3 using the auxiliary system of coordinates $x_{3}, y_{3}$ (ACS3) is shown in Figure 4 .

The course of procedure is as follows:

(i) The type of transition curve is determined.

(ii) The length $l_{3}$ of curve (measured along the curve) is assumed.

(iii) Parametric equations $x_{3}(l), y_{3}(l), l \in\left\langle 0, l_{3}\right\rangle$, are obtained.

The next step is the transformation of transition curve TC3 to the auxiliary system of coordinates $\overline{x_{3}}, \overline{y_{3}}(\overline{\mathrm{ACS} 3})$ whose axes are parallel to the local coordinate system $x$, $y$. The transfer is possible by a right turn of system ACS3 through angle $\delta$. The parametric equations of curve TC 3 in system $\overline{\mathrm{ACS} 3}$ are as follows:

$$
\begin{aligned}
& \overline{x_{3}}(l)=x_{3}(l) \cos \delta-y_{3}(l) \sin \delta, \\
& \overline{y_{3}}(l)=x_{3}(l) \sin \delta+y_{3}(l) \cos \delta,
\end{aligned}
$$

$$
l \in\left\langle-l_{3}, 0\right\rangle .
$$

Inserting the final value of parameter $l$ (i.e., $l=-l_{3}$ ) into (20) gives values $l_{\mathrm{TC} 3}$ and $\Delta y_{\mathrm{TC} 3}$. The value of tangent $s_{K 3}$ at point $K_{3}$ in the $\overline{\mathrm{PUW} 3}$ and LCS systems is

$$
s_{K 3}=\tan \left(-\frac{l_{3}}{2 R_{2}}+\delta\right) \text {. }
$$

The notation of the curve TC3 in the local coordinate system calls for the determination of the position of point $\mathrm{O}_{3}\left(x_{\mathrm{O} 3}, y_{\mathrm{O} 3}\right)$. 


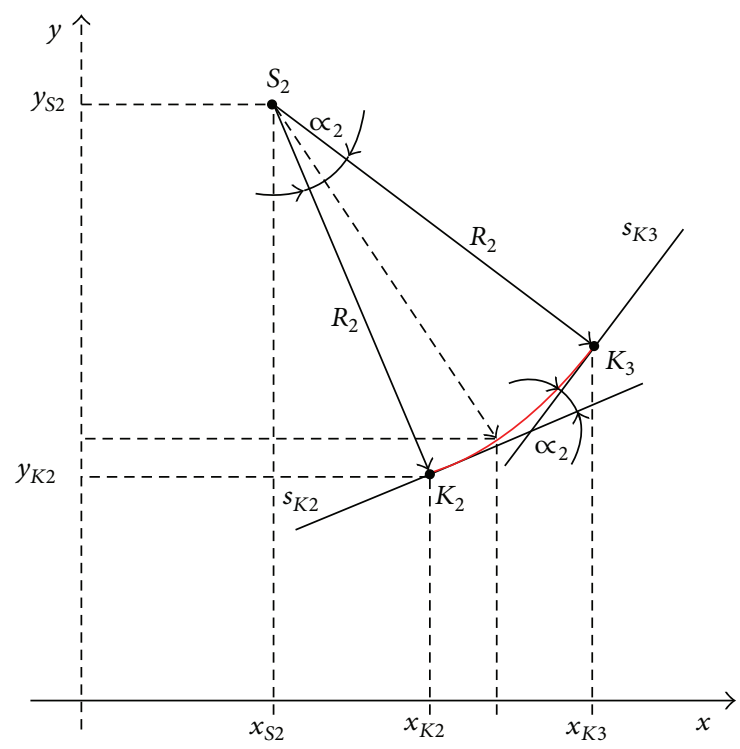

FIGURE 5: Effective scheme of the circular arc CA2 position.

\section{Circular Arc CA2}

An effective scheme of the circular arc CA2 position is shown in Figure 5. At this stage of the design procedure the following data are known: radius $R_{2}$, coordinates of the outset point $K_{2}\left(x_{K 2}, y_{K 2}\right)$, and the tangent values at the start of $s_{K 2}$ and the end of $s_{K 3}$ of arc CA2 (position of the end of arc, i.e., the endpoint coordinates $K_{3}\left(x_{K 3}, y_{K 3}\right)$, is unknown). Thus, to begin with, the coordinates of point $S_{2}\left(x_{S 2}, y_{S 2}\right)$, the mid-point of arc CA2, are determined:

$$
\begin{aligned}
& x_{S 2}=x_{K 2}-\frac{s_{K 2}}{\sqrt{1+s_{K 2}^{2}}} R_{2}, \\
& y_{S 2}=y_{K 2}+\frac{1}{\sqrt{1+s_{K 2}^{2}}} R_{2} .
\end{aligned}
$$

The equation for the circular arc CA2 is as follows:

$$
\begin{aligned}
& y(x)_{\mathrm{CA} 2}=y_{S 2}-\left[R_{2}^{2}-\left(x-x_{S 2}\right)^{2}\right]^{1 / 2}, \\
& \\
& x \in\left\langle x_{K 2}, x_{K 3}\right\rangle .
\end{aligned}
$$

From condition

$$
y\left(x_{K 3}\right)_{\mathrm{CA} 2}^{\prime}=-\frac{x_{K 3}-x_{S 2}}{\left[R_{2}^{2}-\left(x_{K 3}-x_{S 2}\right)^{2}\right]^{1 / 2}}=s_{K 3},
$$

it is possible to determine $x_{K 3}$ and then also $y_{K 3}$ :

$$
\begin{aligned}
& x_{K 3}=x_{S 2}+\frac{s_{K 3}}{\sqrt{1+s_{K 3}^{2}}} R_{2}, \\
& y_{K 3}=y_{S 2}-\frac{1}{\sqrt{1+s_{2}^{2}}} R_{2} .
\end{aligned}
$$

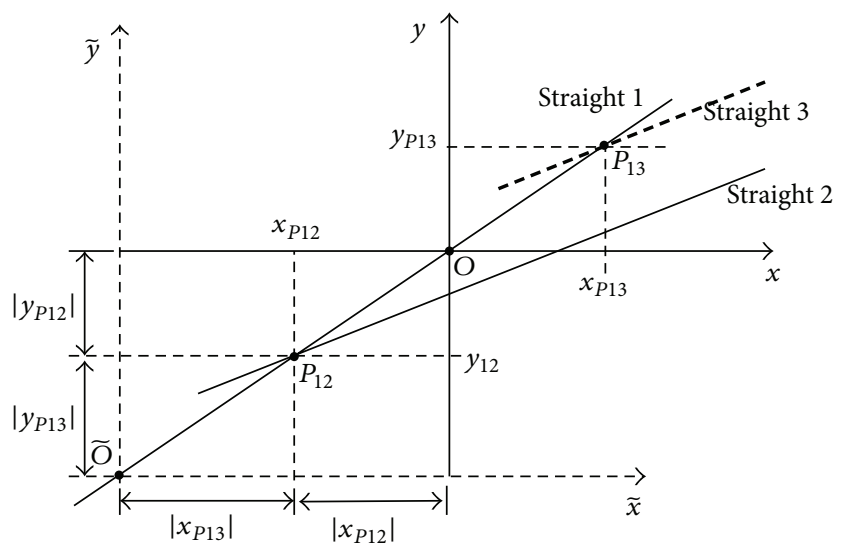

FIGURE 6: Idea of the procedure of correcting the coordinates of the origin of LCS system.

The length of arc CA2 projected on $x$-axis amounts to

$$
l_{\mathrm{CA} 2}=x_{K 3}-x_{K 2},
$$

whereas the turning angle of sense of tangents is

$$
\propto_{2}=\left|\operatorname{atan} s_{K 3}-\operatorname{atan} s_{K 2}\right| \text {. }
$$

Hence the length of the circular arc CA2 (measured along the arc) follows immediately:

$$
l_{R 2}=\propto_{2} R_{2} \text {. }
$$

The knowledge of the position of point $K_{3}\left(x_{K 3}, y_{K 3}\right)$ makes it possible to determine the coordinates of the endpoint $\mathrm{O}_{3}\left(x_{\mathrm{O} 3}, y_{\mathrm{O} 3}\right)$ (Figure 4$)$,

$$
\begin{aligned}
& x_{\mathrm{O} 3}=x_{K 3}+l_{\mathrm{TC} 3}, \\
& y_{\mathrm{O} 3}=y_{K 3}+\Delta y_{\mathrm{TC} 2},
\end{aligned}
$$

and the parametric equations of transition curve TC3:

$$
\begin{array}{r}
x(l)=x_{\mathrm{O} 3}+\overline{x_{3}}(l)=x_{\mathrm{O} 3}+x_{3}(l) \cos \delta-y_{3}(l) \sin \delta, \\
y(l)=y_{\mathrm{O} 3}+\overline{y_{3}}(l)=x_{\mathrm{O} 2}+x_{3}(l) \sin \delta+y_{3}(l) \cos \delta, \\
l \in\left\langle-l_{3}, 0\right\rangle .
\end{array}
$$

\section{Determination of the Right Position of the Origin of the Local Coordinate System}

To transfer the designed geometric system from local coordinate system to the system 2000, one should revise the position of its initial point along Straight 1 . However, in general, point $\mathrm{O}_{3}\left(x_{\mathrm{O} 3}, y_{\mathrm{O} 3}\right)$, denoting the end of the system, will not be lying on Straight 2, but on Straight 3 running parallel to it of equation $y_{(3)}=a_{3}+b_{2} x$ (Figure 6).

In order to determine the corrected coordinates of the outset of LCS system it is necessary to find the coordinates of points intersecting Straight 1 with Straight 2 and with Straight 3. Straight 1 intersects Straight 2 at point $P_{12}$,

$$
x_{P 12}=\frac{a_{2}}{1-b_{2}}=y_{P 12}
$$


and Straight 2 at point $P_{13}$ :

$$
x_{P 13}=\frac{y_{\mathrm{O} 3}-b_{2} x_{\mathrm{O} 3}}{1-b_{2}}=y_{P 13} .
$$

It is intended to shift the start of LCS system (point $O$ ) along Straight 1 to a new position at point $\widetilde{O}$ in such a way that $\tilde{x}_{P 12}=x_{P 13}$ within $\tilde{x}, \tilde{y}$ system (and, of course, $\tilde{y}_{P 12}=y_{P 13}$ ) (Figure 6). This leads to the following general equation:

$$
x_{\widetilde{\mathrm{O}}}=y_{\widetilde{\mathrm{O}}}=x_{P 12}-x_{P 13} .
$$

The revised origin of the local coordinate system in system 2000 has the following coordinates:

$$
\begin{aligned}
Y_{\widetilde{O}} & =Y_{O}+x_{\widetilde{O}} \cos \beta-y_{\widetilde{O}} \sin \beta, \\
X_{\widetilde{O}} & =X_{O}+x_{\widetilde{O}} \sin \beta+y_{\widetilde{O}} \cos \beta .
\end{aligned}
$$

The transference of the designed geometric system from LCS system to system 2000 is carried out by making use of the following relations [22]:

$$
\begin{aligned}
& Y=Y_{\widetilde{O}}+x \cos \beta-y \sin \beta, \\
& X=X_{\widetilde{O}}+x \sin \beta+y \cos \beta .
\end{aligned}
$$

\section{Calculation Examples}

Example 1 (typical case-connection of straights of proximate parallelisms). Consider the following:

Straight 1:

$$
\begin{aligned}
& X_{1}=27878029,36485-3,365805196 Y, \\
& \varphi_{1}=1,859595714 \mathrm{rad}, \\
& \beta=1,074197551 \mathrm{rad} .
\end{aligned}
$$

Straight 2:

$$
\begin{aligned}
& X_{2}=35851110,27727-4,592528563 Y, \\
& \varphi_{2}=1,785194693 \mathrm{rad} .
\end{aligned}
$$

Assumed Starting Point:

$$
\begin{aligned}
& Y_{O}=6498745,04911 \mathrm{~m}, \\
& X_{O}=6004519,50986 \mathrm{~m} .
\end{aligned}
$$

\section{Design Data:}

$$
\begin{aligned}
& v=90 \mathrm{~km} / \mathrm{h}, \\
& l_{1}=90 \mathrm{~m} \text { (clothoid), } \\
& \psi_{1}=0,17 \mathrm{~m} / \mathrm{s}^{3}, \\
& f_{1}=27,8 \mathrm{~mm} / \mathrm{s}, \\
& R_{1}=500 \mathrm{~m}\left(l_{R 1}=150 \mathrm{~m}\right), \\
& h_{1}=100 \mathrm{~mm}, \\
& a_{m 1}=0,60 \mathrm{~m} / \mathrm{s}^{2}, \\
& l_{2}=160 \mathrm{~m}(\text { linear curvature }), \\
& \psi_{2}=0,18 \mathrm{~m} / \mathrm{s}^{3},
\end{aligned}
$$

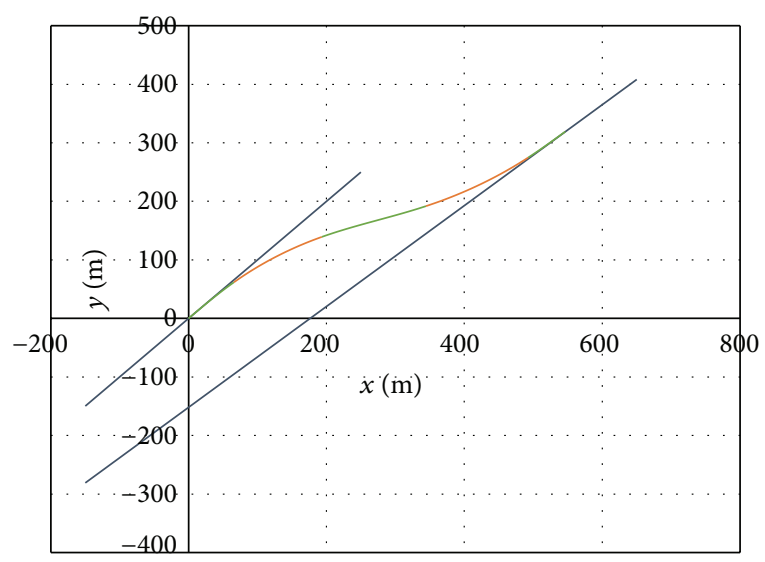

Figure 7: Visualization of the designed geometric system (Example 1).

$$
\begin{aligned}
& f_{2}=26,6 \mathrm{~mm} / \mathrm{s}, \\
& R_{2}=600 \mathrm{~m}\left(l_{R 2}=170,359 \mathrm{~m}\right), \\
& h_{2}=70 \mathrm{~mm}, \\
& a_{m 2}=0,58 \mathrm{~m} / \mathrm{s}^{2}, \\
& l_{3}=70 \mathrm{~m} \text { (clothoid), } \\
& \psi_{3}=0,21 \mathrm{~m} / \mathrm{s}^{3}, \\
& f_{3}=25,0 \mathrm{~mm} / \mathrm{s},
\end{aligned}
$$

where $v$ is speed of trains, $h_{1}$ is cant along arc CA1, $h_{2}$ is cant along arc CA2, $a_{m 1}$ is unbalanced acceleration along arc CA1, $a_{m 2}$ is unbalanced acceleration along arc CA2, $\psi_{1}$ is speed of acceleration change on transition curve TCl, $\psi_{2}$ is speed of acceleration change on transition curve TC2, $\psi_{3}$ is speed of acceleration change on transition curve TC $3, f_{1}$ is speed of lifting the wheel at the cant transition of curve TC1, $f_{2}$ is speed of lifting the wheel at the cant transition of curve TC2, and $f_{3}$ is speed of lifting the wheel at the cant transition of curve TC3.

Correction of the Initial Position of LCS System:

$$
\begin{aligned}
& Y_{\widetilde{\mathrm{O}}}=6,499053,754 \mathrm{~m}, \\
& X_{\widetilde{\mathrm{O}}}=6003480,469 \mathrm{~m} .
\end{aligned}
$$

Straight 1:

$$
y_{(1)}=x .
$$

Straight 2:

$$
y_{(2)}=-257,909+0,86126 x .
$$

Figure 7 presents the visualization of the designed geometrical layout from Example 1. In Table 1 characteristics of the principal points of the geometrical layout are given. The combination of two straight lines close to the parallelism is carried out on the length of $640,359 \mathrm{~m}$. The length of the circular arc CA2 closing the entire layout is $l_{R 2}=170,359 \mathrm{~m}$. It should be noted that the correction of the local coordinate system beginning is significant, abscissa $Y$ about $308 \mathrm{~m}$ and ordinate $X$ about $1039 \mathrm{~m}$. 
TABLE 1: Characteristic of the principal points of the geometric system (Example 1).

\begin{tabular}{lcccc}
\hline Point & $x(\mathrm{~m})$ & $y(\mathrm{~m})$ & $s$ & $\Theta(\mathrm{rad})$ \\
\hline$O$ & 0 & 0 & 1,000 & 0,7854 \\
$K_{1}$ & 65,496 & 61,680 & 0,834 & 0,6954 \\
$O_{2}$ & 193,254 & 139,202 & 0,417 & 0,3954 \\
$K_{2}$ & 344,580 & 191,579 & 0,386 & 0,3687 \\
$K_{3}$ & 492,704 & 274,569 & 0,764 & 0,6527 \\
$O_{3}$ & 546,613 & 319,204 & 0,861 & 0,7110 \\
\hline
\end{tabular}

The practical applicability to the solution presented in Example 1 (Figure 7) cannot raise any doubts. Taking into consideration only the calculation technique, some problems arise when parallel straights are joined, since it is not possible under such circumstances to make a direct correction of the local coordinate system position. However, the computeraided calculations by the use of the mentioned algorithm make it possible to solve this problem easily.

Example 2 (a case of more diversified gradients of both the straights). Data relating to Straight 1 , of the assumed outset point, and the design characteristic are shown as in Example 1 (where the obtained length $l_{R 2}=80,350 \mathrm{~m}$ ).

Straight 2:

$$
\begin{aligned}
& X_{2}=106803989,47315-15,51050124 Y, \\
& \varphi_{2}=1,635179667 \mathrm{rad} .
\end{aligned}
$$

Correction of the Initial Position of LCS System:

$$
\begin{aligned}
& Y_{\widetilde{O}}=6498784,841 \mathrm{~m}, \\
& X_{\widetilde{\mathrm{O}}}=6004385,577 \mathrm{~m} .
\end{aligned}
$$

Straight 1:

$$
y_{(1)}=x .
$$

Straight 2:

$$
y_{(2)}=-14,706+0,62832 x .
$$

Visualization of the designed geometrical layout from Example 2 is presented in Figure 8. In Table 2 characteristics of the principal points of the geometrical layout are given. The combination of two straight lines is carried out on the length of $550,35 \mathrm{~m}$. The length of the circular arc CA2 closing the entire layout is $l_{R 2}=80,35 \mathrm{~m}$. The correction of the local coordinate system beginning is relatively small, abscissa $Y$ about $40 \mathrm{~m}$ and ordinate $X-140 \mathrm{~m}$.

However, at first glance the solution presented in Example 2 (Figure 8) may raise some doubts. It may appear that inverse curves are of no use in this situation and both the straights should be connected in an elementary way using a circular arc with transition curves. However, in the design process one must take account of a need to pass over a field obstacle and then the inverse curves can become a sensible solution. As can be seen, the inverse curves may provide an alternative even to such an elementary geometric problem, which is joining two straights by means of a circular arc.
TABLE 2: Characteristic of the principal points of the geometric system (Example 2).

\begin{tabular}{lcccc}
\hline Point & $x(\mathrm{~m})$ & $y(\mathrm{~m})$ & $s$ & $\Theta(\mathrm{rad})$ \\
\hline$O$ & 0 & 0 & 1,000 & 0,7854 \\
$K_{1}$ & 65,496 & 61,680 & 0,834 & 0,6954 \\
$O_{2}$ & 193,254 & 139,202 & 0,417 & 0,3954 \\
$K_{2}$ & 344,580 & 191,579 & 0,386 & 0,3687 \\
$K_{3}$ & 417,370 & 225,465 & 0,550 & 0,5026 \\
$O_{3}$ & 477,345 & 261,541 & 0,628 & 0,5608 \\
\hline
\end{tabular}

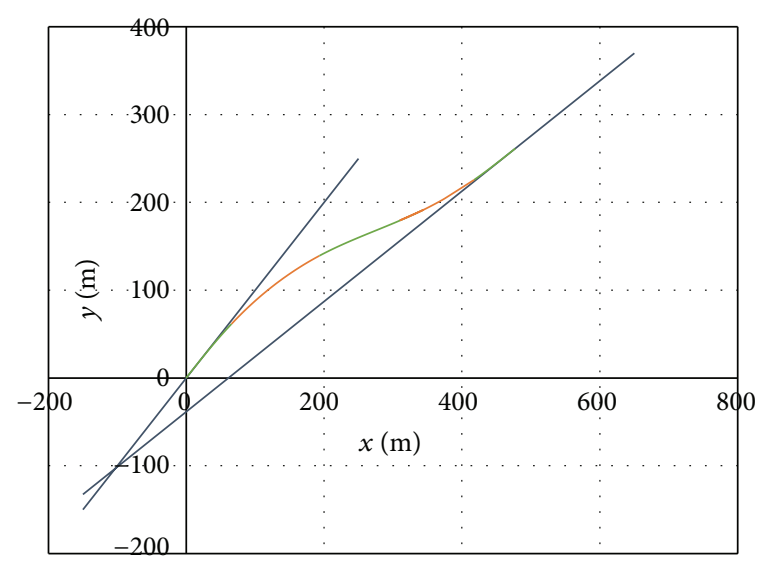

Figure 8: Visualization of the designed geometric system (Example 2).

The calculation examples show the correctness of the developed method as well as the opportunity to apply it using elementary way, that is, calculation sheets. However, this universal algorithm can be easily applied to the computer software which will allow generating, in an automatic way, by changing the radii of the arcs and the type and length of the transition curves, another geometrical layout. Then, the choice of the most beneficial variant from the point of obtained trains velocities while minimizing the track axis offsets will be held using the optimization techniques. The current designing methods do not provide such opportunities.

\section{Summing-Up}

(i) The application of Mobile Satellite Measurements with antennas installed on a moving rail vehicle makes it possible to reconstruct the track axis in an absolute reference system. This creates completely new potentials in the range of rail track geometric shaping. Under conditions of the created situation there arises a necessity for working out some new design methods.

(ii) This paper presents one method more (following the studies in $[18,19])$ relating to the design of a region of railway track direction change appropriate for Mobile Satellite Measurement technique. The method may appear to be of particular applicability if both straights of the track direction cannot be connected in an elementary way by the use of a circular arc with transition curves; this also concerns 
the use of the compound curve. Such a situation occurs when the connected straights indicate values of the inclination coefficient which are very close to each other and intersect at a distant point (they may also run parallel). In such a situation the only solution is to introduce to the geometric system two circular arcs of opposite signs of curvature, that is, to apply an inverse curve.

(iii) The presented conception of the design procedure relating to the region covering the track direction change offers an opportunity to find an analytical solution, by the use of appropriate mathematical formulae, being most friendly in practical application. The design procedure is of a universal nature and creates a possibility for arbitrary acceptance of lengths and radii of circular arcs and differentiation of the type and lengths of the applied transition curves.

(iv) The effects of the application of the analyzed design method have been illustrated by exact calculation examples. Its practical applicability cannot cause any doubts. Simultaneously attention has been concentrated on the fact that the inverse curves may provide an alternative even for such an elementary geometric problem which is the connection of two straights by using a circular arc. In order to implement the presented procedure it will be indispensable to work out in the near future an appropriate computer-aided technique. The computer software will allow generating automatically additional geometrical layouts. The choice of the best solution will be held in the field of optimization. The criteria of optimizations are the maximum value of the velocity and minimizing the track axis offsets. The current designing methods do not provide such opportunities.

\section{Competing Interests}

The author declares that he has no competing interests.

\section{References}

[1] G. Seeber, Satellite Geodesy: Foundations, Methods and Applications, Walter de Gruyter, Berlin, Germany, 1993.

[2] B. W. Parkinson, J. J. Spilker Jr., P. Axelrad, and P. Enge, Global Positioning System: Theory and Applications, Volume II, American Institute of Aeronautics and Astronautics, Reston, Va, USA, 1996.

[3] M. Ferguson, GPS Land Navigation, Glassford, Spokane, Wash, USA, 1997.

[4] J. Bosy, W. Graszka, and M. Leonczyk, "ASG-EUPOS-a multifunctional precise satellite positioning system in Poland," European Journal of Navigation, vol. 5, no. 4, pp. 2-6, 2007.

[5] A. Leick, L. Rapoport, and D. Tatarnikov, GPS Satellite Surveying, John Wiley \& Sons, Hoboken, NJ, USA, 4th edition, 2015.

[6] W. Koc and C. Specht, "Application of the Polish active GNSS geodetic network for surveying and design of the railroad," in Proceedings of the 1st International Conference on Road and Rail Infrastructure (CETRA '10), Opatija, Croatia, May 2010.

[7] C. Specht, A. Nowak, W. Koc, and A. Jurkowska, "Application of the Polish Active Geodetic Network for railway track determination," in Transport Systems and Processes-Marine Navigation and Safety of Sea Transportation, pp. 77-81, CRC Press, London, UK, 2011.
[8] T. Szwilski, R. Begley, P. Dailey, and Z. Sheng, "Determining rail track movement trajectories and alignment using HADGPS," in Proceedings of the AREMA Annual Conference, Chicago, Ill, USA, October 2003.

[9] Council of Ministers of 15 October 2012 on National spatial reference system, Dziennik Ustaw, pos. 1247, 2012 (Polish).

[10] W. Koc and C. Specht, "Selected problems of determining the course of railway routes by use of GPS network solution," Archives of Transport, vol. 23, no. 3, pp. 303-320, 2011.

[11] W. Koc, C. Specht, and P. Chrostowski, "Finding deformation of the stright rail track by GNSS measurements," Annual of Navigation, vol. 19, no. 1, pp. 91-104, 2012.

[12] W. Koc, C. Specht, P. Chrostowski, and K. Palikowska, "The accuracy assessment of determining the axis of railway track basing on the satellite surveying," Archives of Transport, vol. 24, no. 3, pp. 307-320, 2012.

[13] W. Koc, C. Specht, and P. Chrostowski, "The application effects of continuous satellite measurements of railway lines," in Proceedings of the 12th International Conference \& Exhibition Railway Engineering, Railway Operation Section, London, UK, July 2013.

[14] M. Lindahl, Track Geometry for High Speed Railways, KTH, Stockholm, Sweden, 2001.

[15] M. H. Letts, "Track geometry design testing for transit applications," Final Report for Transit IDEA Project 41, Transportation Research Board of the National Academies, Washington, DC, USA, 2007.

[16] P. Lautala and T. Dick, Railway Alignment Design and Geometry, AREMA, Lanham, Md, USA, 2010.

[17] S. Hodas, "Design of railway track for speed and high-speed railways," Proceedia Engineering, vol. 91, pp. 256-261, 2014.

[18] W. Koc, "Design of rail-track geometric systems by satellite measurement," Journal of Transportation Engineering, vol. 138, no. 1, pp. 114-122, 2012.

[19] W. Koc, "Design of compound curves adapted to the satellite measurements," Archives of Transport, vol. 34, no. 2, pp. 37-49, 2015.

[20] W. Koc and P. Chrostowski, "Computer-aided design of railroad horizontal arc areas in adapting to satellite measurements," Journal of Transportation Engineering, vol. 140, no. 3, Article ID 04013017, 2014.

[21] http://www.innotrack.net.

[22] G. A. Korn and T. M. Korn, Mathematical Handbook for Scientists and Engineers, McGraw-Hill, New York, NY, USA, 1968.

[23] W. Koc, "Analytical method of modelling the geometric system of communication route," Mathematical Problems in Engineering, vol. 2014, Article ID 679817, 13 pages, 2014. 


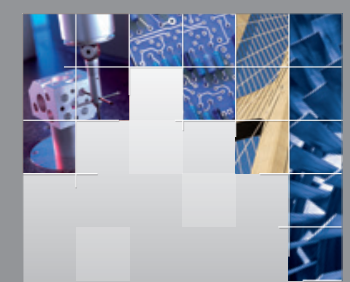

\section{Enfincering}
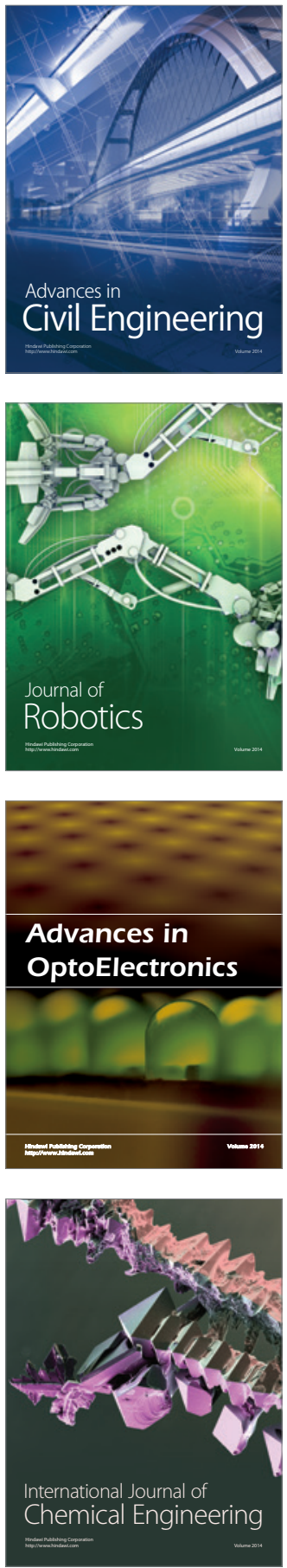

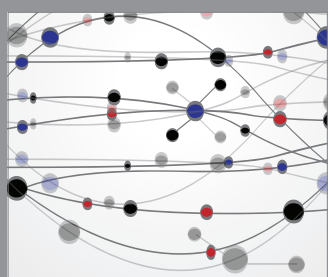

The Scientific World Journal

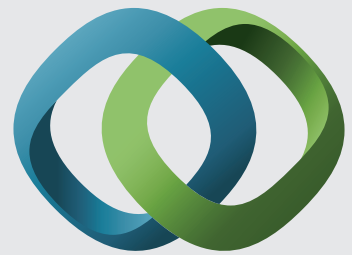

\section{Hindawi}

Submit your manuscripts at

http://www.hindawi.com
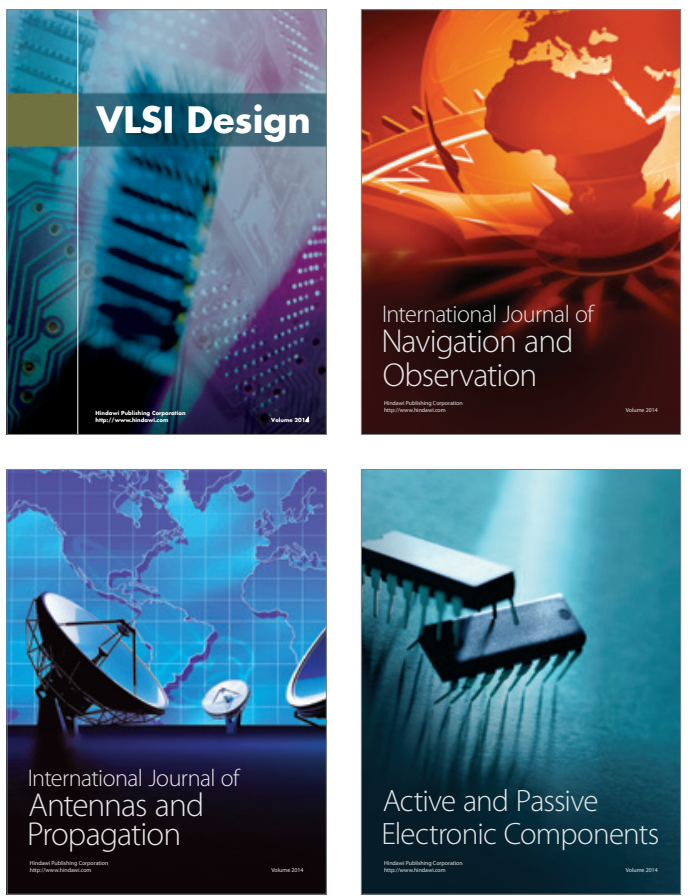
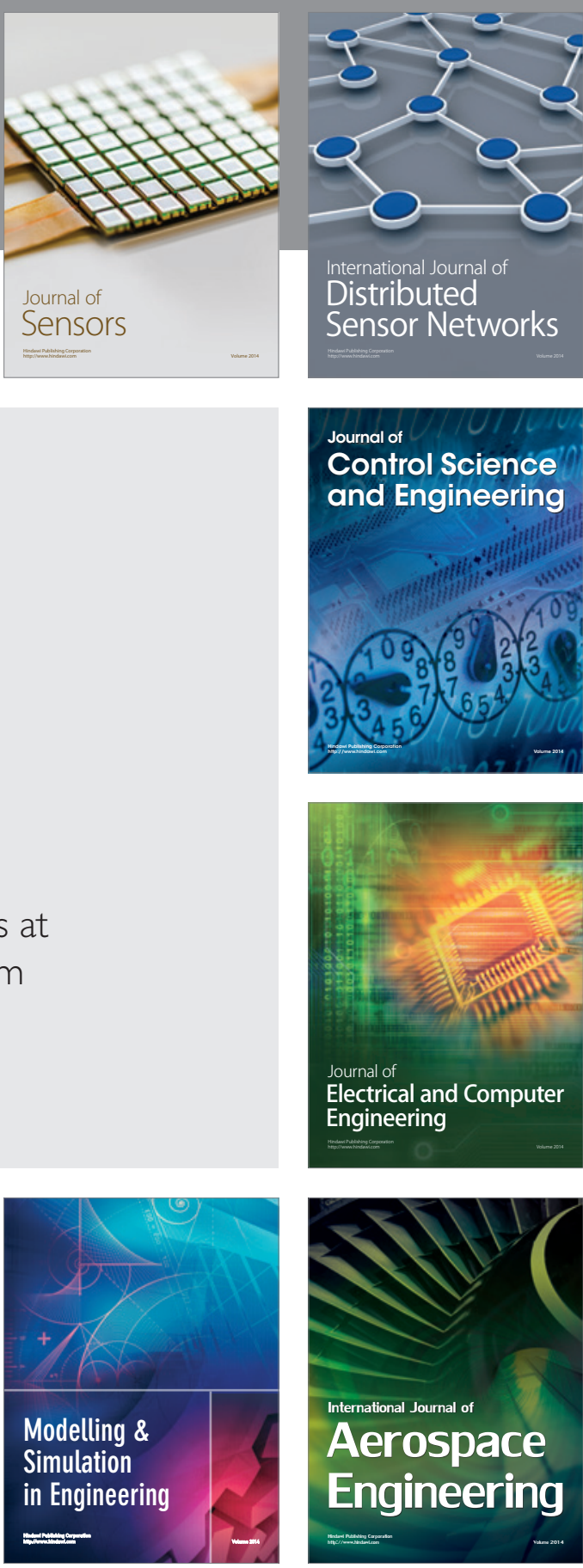

International Journal of

Distributed

Sensor Networks

Journal of

Control Science

and Engineering
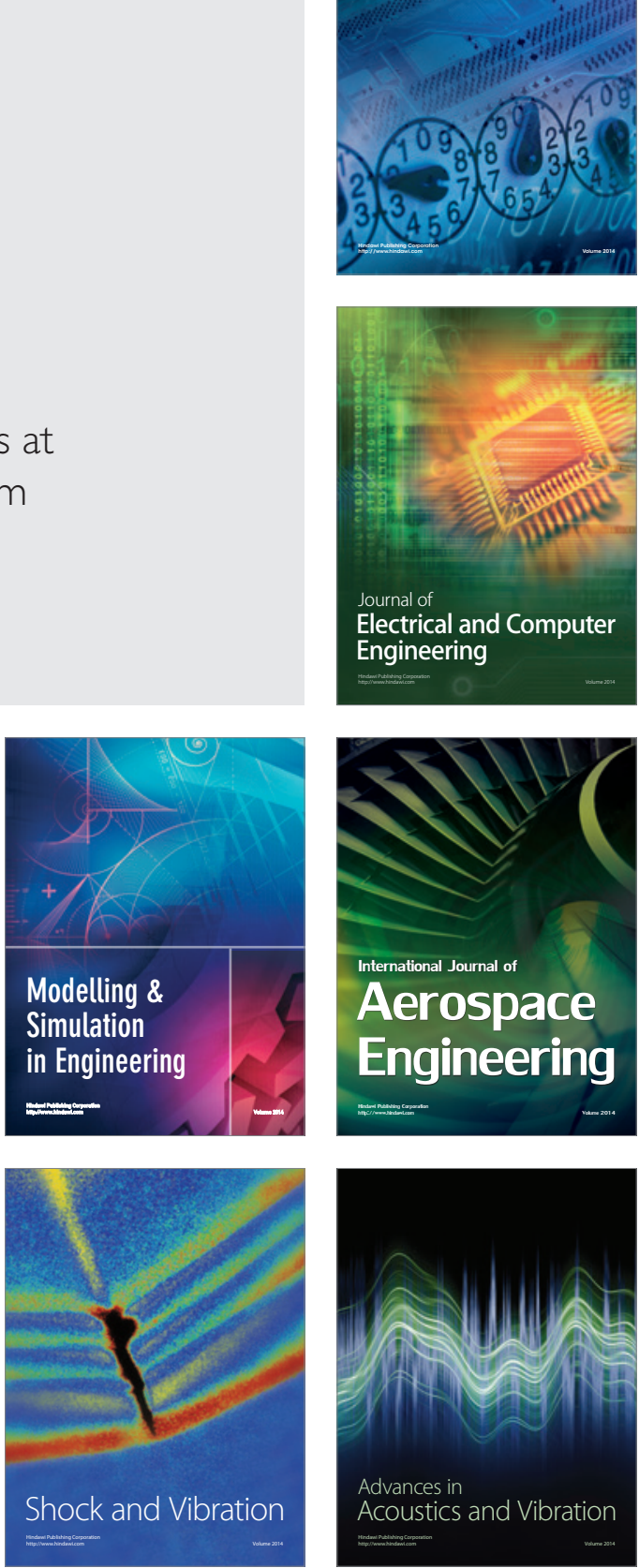